\title{
Expression of Fas Receptor and Fas Ligand in Eczematous Dermatitis
}

\author{
Hanan Rabea Nada ${ }^{1}$, Ahmed M. Nada ${ }^{1}$, Laila A. Rashed ${ }^{2}$, Mira M. Abdel Halim ${ }^{3}$ and Maha Fathy \\ Elmasry (D) ${ }^{1, *}$ \\ ${ }^{1}$ Dermatology Department, Faculty of Medicine, Cairo University, Cairo, Egypt \\ ${ }^{2}$ Medical Biochemistry and Molecular Biology Department, Faculty of Medicine, Cairo University, Cairo, Egypt \\ ${ }^{3}$ Cairo, Egypt \\ "Corresponding author: Dermatology Department, Faculty of Medicine, Cairo University, Cairo, Egypt. Tel: +20-1222633740, Email: mahafathy1214@gmail.com \\ Received 2019 September 13; Revised 2019 December 13; Accepted 2019 December 15.
}

\begin{abstract}
Background: The principal role of epidermal keratinocytes (KCs) in inducing and maintaining eczematous dermatitis has been rather neglected.

Objectives: The study aimed to assess the expression of Fas receptor(FasR)and Fas ligand(FasL) in the skin of eczematous dermatitis patients to examine T-cell mediated KC apoptosis.

Methods: The population included 35 patients with eczematous dermatitis, as well as 15 age- and sex-matched healthy controls. The cases group included 13 eczema, five atopic dermatitis (AD) and 17 contact dermatitis (CD) patients. Tissue levels of FasR and FasL were estimated using a quantitative real time-polymerase chain reaction.

Results: FasR and FasL were upregulated in the cases group compared to the control group $(\mathrm{P}<0.001)$. Moreover, FasR and FasL were upregulated in the eczema cases compared to the cases with $\mathrm{CD}$ and $\mathrm{AD}(\mathrm{P}=0.001)$. On the contrary, there was downregulation of FasR and FasL in the $\mathrm{CD}$ cases in comparison to the cases having eczema and $\mathrm{AD}(\mathrm{P}=0.001)$.

Conclusions: The study concluded that the upregulation of FasR and FasL may be involved in the pathogenesis of eczema through induction of KC apoptosis. This might help in the future use of anti-apoptotic therapy for prevention and treatment of eczematous dermatitis.
\end{abstract}

Keywords: Apoptosis, Eczema, Fas Ligand, Fas Receptor

\section{Background}

Eczematous dermatitis constitutes a large proportion of inflammatory dermatological diseases characterized by a typical morphological reaction pattern $(1,2)$. The classification of eczematous dermatoses is greatly variable. Some classifications depend on aetiological factors in case they are well-known [e.g., contact dermatitis (CD)], some depend on the morphological pattern (e.g., nummular eczema), and others depend on regional variants (e.g., hand eczema and napkin dermatitis) (3). Another approach for classification of eczematous dermatoses is to divide different forms of eczema into endogenous and exogenous variants. The particular value of these classifications for the clinician is that they imply an approach to management (4).

Eczematous dermatitis usually has three phases: acute, subacute, and chronic phases. In the acute phase, the histological picture is dominated by spongiosis, an intercellular epidermal edema which leads to stretching and even- tual rupture of intercellular attachments with vesicle formation. In the subacute stage, spongiosis and vesiculation diminish, and increasing acanthosis is associated with formation of a parakeratotic horny layer. Moreover, there is significant acanthosis with hyperkeratosis and minimal parakeratosis in the chronic phase (5).

The pathogenesis of spongiosis is not fully understood, and many hypotheses have been suggested in this regard. The process of spongiosis entails two events: detachment of keratinocytes (KCs) from each other and fluid influx between the detached cells because of the osmotic pressure gradient. However, no one can explain which of these two events begins first (6).

Fas receptor [apoptosis antigen 1(Apo-1) (APT 1)/cluster of differentiation 95 (CD95)/tumor necrosis factor superfamily 6 (TNFRSF6)] is a transmembrane receptor (7) that belongs to the death receptor family. It is a subgroup of the tumor necrosis factor (TNF)/nerve growth factor (NGF) receptor superfamily (8) and acts as the target of cell deathinducing antibodies (9). In addition to its apoptotic func- 
tion, Fas receptor (FasR) has other cellular responses including migration, invasion, inflammation, and proliferation (10).

\section{Objectives}

We aimed in this study to assess the expression of FasR and Fas ligand (FasL) in the lesional skin of eczematous dermatitis [including eczema, atopic dermatitis (AD), and contact dermatitis (CD)] to allow the study of T-cell mediated KC apoptosis.

\section{Methods}

The study was conducted in a dermatology outpatient clinic after approval of the Dermatology Research Ethical Committee (Protocol number: 20/2014). The population included 35 patients with eczematous dermatitis, as well as 15 sex- and age-matched healthy controls, during the time interval from December 2014 to April 2015. Written informed consents were obtained from all the participants.

\subsection{Study Design}

This case-control study included two groups:

\subsubsection{Cases Group}

This group included 35 patients with eczematous dermatitis, of whom 13 had eczema, five had $\mathrm{AD}$, and 17 had $\mathrm{CD}$. Inclusion criteria were patients of both sexes with acute and subacute eczematous dermatitis taking no systemic or topical treatment for at least one month prior to inclusion into the study. Moreover, exclusion criteria included chronic eczematous dermatitis cases or any case taking topical or systemic therapy less than one month prior to inclusion into the study.

Written informed consents along with detailed information on medical history were collected from all the patients. Further, they underwent full clinical examination and skin biopsy. Clinical assessment was performed to determine the extent of eczema and the disease severity using the eczema area and severity index (EASI) (11).

\subsubsection{Control Group}

Fifteen age- and sex-matched healthy volunteers were included as a control group. All the recruits were with no history of acute or chronic dermatological or systemic diseases including renal and liver diseases or malnutrition. All the control group participants submitted written informed consents and underwent skin biopsy.

\subsection{Skin Biopsy}

Four millimeter punch biopsies were taken from the lesional skin of the studied patients and the normal controls. Local anesthesia was injected around the biopsy site. The skin biopsies were stored in an empty test tube at $-70^{\circ} \mathrm{C}$ for quantitative real time-polymerase chain reaction (qRTPCR).

\subsection{Laboratory Methods}

\subsection{1. qRT-PCR Protocol}

Principle: A typical workflow of qRT-PCR for gene expression measurement involves RNA isolation, reverse transcription, qRT-PCR assay development, qRT-PCR experiment, and data analysis.

Reagents for RNA extraction: They include total RNA purification kit (Jena Bioscience) consisting of lysis buffer, spin columns, activated buffer, primary washing buffer (96\% - 99\% ethanol was added before use), 2 millimeter tubes for collection, secondary washing buffer (96\% - 99\% ethanol was added before use), and elution buffer.

Reagents for cDNA synthesis: They include Maxime RT PreMix kit (iNtRON Biotechnology) consisting of Maxime RT PreMix (Oligo dT Primer) 96 tubes.

Reagents for qRT-PCR: They include SibirHot Master Mix (qRT-PCR $2 \times)($ BIORON GmbH) consisting of qRT-PCR.

\subsubsection{Assay Procedure}

Ribonucleic acid (RNA) extraction from skin tissue biopsies: sample preparation and cell lysis: A volume of 20 to $50 \mathrm{mg}$ of fresh skin biopsy was collected in a microcentrifugation tube. Thereafter, $300 \mu$ L lysis buffer was added and then homogenization was performed using a pellet pestle; an apparatus operated by hand. Afterwards, a further $200 \mu \mathrm{L}$ lysis buffer was added using a vortex for 15 - 30 seconds. Centrifugation was performed at 10,000 $\mathrm{g}$ for 10 minutes.

If debris remained in the supernatant, $500 \mu \mathrm{L}$ chloroform was added followed by using a vortex for 15 - 30 seconds. Next, 10,000 g centrifugation was applied for $10 \mathrm{~min}$ utes. The supernatant was then transferred into microcentrifuge tubes.

3.3.2.1. Column Activation Followed by Column Loading as Well as Primary and Secondary Column Washing

RNA elution: A spin column was placed into a microcentrifuge tube free of ribonuclease. Then, 40 - $50 \mu \mathrm{L}$ elution buffer was added to the middle of the column membrane. This was followed by incubation at room temperature for 1 minute. Afterwards, 10,000 g centrifugation was 
performed in 1 minute for RNA elution. RNA was stored at $-20^{\circ}$ to $-80^{\circ} \mathrm{C}$.

Reverse transcription into cDNA: Template RNA and distilled water were added into Maxime RT PreMix tubes (Oligo dT or random primer) to a total volume of $20 \mu \mathrm{l}$. The clear pellet was dissolved by pipetting. AcDNA synthesis reaction was performed as follows using a PCR machine. The above reactant was diluted by adding 20-50 $\mu \mathrm{l}$ sterile water into a tube containing cDNA obtained at RT.

DNA amplification: A vortex was used for the samples and briefly centrifuged to collect all drops to the bottom of the tube. The samples were placed in a thermocycler and a PCR program was initiated. Sequences of the primers used for real-time PCR were as follows:

FasR: forward primer: 5'-CGTGAAACCGACAACAACTG-3'

Reverse primer: 5'-TTTTCGTTCACCAGGCTGAC-3'

FasL: forward primer: 5'-TCTGGTTGAATGGGGTTAG-3'

Reverse primer: 5'TAAGGCTGTGGTTGGTGAAC-3'

$\beta$-actin: $\quad$ forward primer:

5'-

ATGAGCCCCAGCCTTCTCCAT-3'

Reverse primer: 5'-CCAGCCGAGCCACATCGCTC-3'

\subsection{Statistical Methods}

Results were expressed as mean \pm standard deviation (SD) or number (\%). Comparison was performed between the categorical data sets using the $\chi^{2}$ test. According to the test of normality, comparison was performed between the two groups in terms of different variables using the Mann-Whitney U test. Further, comparison between different variables was performed using the Kruskal-Wallis test. $P$ value $\leq 0.05$ was considered significant and less than 0.01 was considered highly significant. Moreover, correlations between FasR and FasL and different variables were measured using the Spearman's rho correlation test. Data analysis was performed using Statistical Package for Social Sciences (SPSS) version 19 for Windows.

\section{Results}

4.1. Baseline Characteristics and Clinical Data of Studied Groups

This study was conducted on 35 patients with eczematous dermatitis and 15 normal subjects serving as controls.

\subsubsection{Cases Group}

The patients were 23 males (65.7\%) and 12 females (24.3\%) aging from 7 to 60 years with the mean of $31.33 \pm$ 13.880 years (Table 1 ). The disease lasted from 7 - 42 days with the mean of $25.97 \pm 8.60$ days. EASI scores of the patients ranged from 1 to 35 with the mean of $7.46 \pm 9.05$. The sites of lesions were localized in 27 cases (77.1\%) and generalized in eight cases (22.8\%). Of the 35 patients, 13 were eczema cases (37.1\%), five were AD cases (14.2\%), and 17 were CD cases (48.5\%). The stages were acute in six cases (17.1\%), subacute in 20 cases (57.1\%), and acute exacerbation on top of chronic in nine cases (8.57\%).

Table 1. The Correlation Between Fas Receptor and Fas Ligand with the Different Studied Variables in the Cases Group ${ }^{\mathrm{a}, \mathrm{b}}$

\begin{tabular}{lcclc}
\hline & \multicolumn{2}{c}{ Fas Receptor } & \multicolumn{2}{c}{ Fas Ligand } \\
\cline { 2 - 5 } & $\mathbf{r}$ & $\mathbf{P}$ & $\mathbf{r}$ & $\mathbf{P}$ \\
\hline Age & -0.159 & 0.362 & -0.264 & 0.125 \\
Duration & 0.327 & 0.055 & 0.327 & 0.055 \\
\hline Redness & -0.179 & 0.305 & -0.143 & 0.414 \\
Thickness & 0.180 & 0.301 & 0.082 & 0.638 \\
\hline Scratching & 0.227 & 0.189 & 0.142 & 0.415 \\
\hline EASI score & 0.085 & 0.626 & 0.032 & 0.855 \\
\hline $\begin{array}{l}\text { a } \mathrm{r}=\text { Spearman's rho correlation coefficient. } \\
\mathrm{b} P \text { (significant if }<0.05) .\end{array}$ & & & \\
\hline
\end{tabular}

\subsubsection{Control Group}

The controls were seven males (46.7\%) and eight females (53.3\%) aging from 17 to 53 years with the mean of $29.73 \pm 11.548$ years.

\subsection{Comparison Between Studied Groups}

Comparison of the patients and the controls yielded no statistically significant differences in terms of age and sex $(\mathrm{P}>0.05)$.

4.3. Comparison of Fas Receptor and Fas Ligand in Studied Groups

The FasR mean was statistically higher in the cases group (8.23 \pm 3.59$)$ than in the control group (1.14 \pm 0.29$)$ (P $<0.001)$. Similarly, the FasL mean was higher in the cases $(5.54 \pm 3.11)$ than in the controls $(1.07 \pm 0.13)(\mathrm{P}<0.001)$ (Figure 1).

\subsection{FasR and FasL in Relation to Other Variables}

4.4.1. Sex

Comparison of FasR and FasL showed no statistically significant differences between the male and female patients in the cases group ( $\mathrm{P}>0.05)$. Similarly, no statistical differences were observed between the male and female controls in terms of FasR and FasL $(\mathrm{P}>0.05)$. 


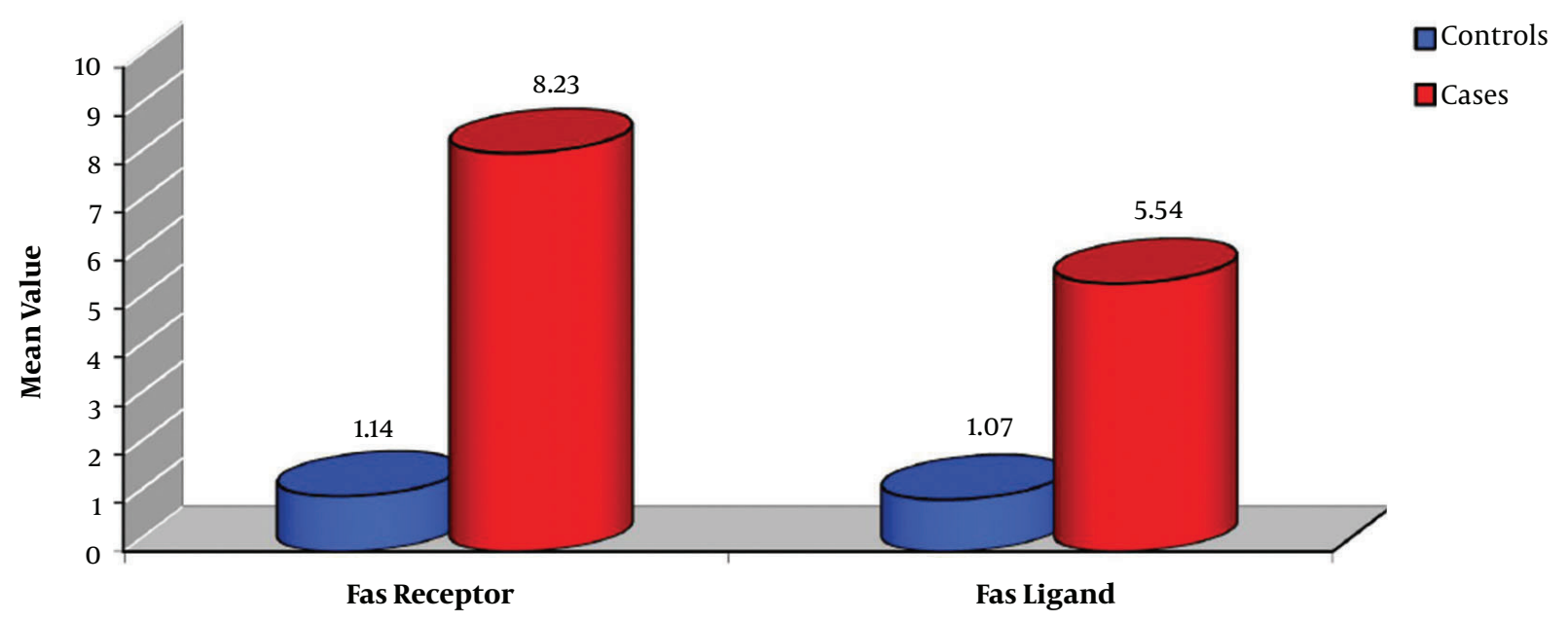

Figure 1. The comparison between the two studied groups in terms of the mean values of Fas receptor and Fas ligand

\subsubsection{Other Variables}

No statistically significant correlations were found between FasR and FasL with the age of the patients, the disease duration, the degree of redness of the lesions, the thickness of the lesions, the scratching of the lesions, and the EASI score (Table 1).

\subsubsection{Stage of Disease}

The stage of the disease was shown to be statistically uncorrelated with FasR and FasL (Table 2).

\subsubsection{Site of Disease}

No statistically significant correlations were also observed between FasR and FasL with the site of the disease $(\mathrm{P}>0.05)$. Accordingly, the FasR mean was $8.13 \pm 3.57$ in localized cases (27 cases) and $8.60 \pm 3.87$ in generalized cases (eight cases). Moreover, the FasL mean was $5.37 \pm 3.17$ in localized cases and $6.13 \pm 3.05$ in generalized cases.

\subsubsection{Type of Eczematous Dermatitis}

The FasR mean was statistically lower in the patients with $\mathrm{CD}(6.26 \pm 2.24)$ than in those with eczema and $\mathrm{AD}$ $(10.10 \pm 3.67)(P=0.001)$. Further, the FasL mean was statistically lower in the $\mathrm{CD}$ patients $(3.75 \pm 1.21)$ than in the eczema and AD patients $(7.24 \pm 3.43)(\mathrm{P}=0.001)$ (Table 3$)$.

In addition, the FasR mean was higher in patients with eczema (11.30 \pm 3.16$)$ than in those with $\mathrm{CD}$ and $\mathrm{AD}(6.42 \pm$ 2.43) $(\mathrm{P}=0.001)$. In a similar vein, the FasL mean was higher in the patients with eczema $(8.19 \pm 3.37)$ than in the $C D$ and AD patients $(8.19 \pm 3.37)(\mathrm{P}=0.001)$ (Table 3$)$.
Comparison of FasR and FasL revealed no statistically significant differences between the patients with $\mathrm{AD}$ and those with $\mathrm{CD}$ and eczema $(\mathrm{P}>0.05)$ (Table 3 ).

\section{Discussion}

The pathogenesis of the hallmark sign of acute eczema, i.e. spongiosis, is not yet completely recognized. Numerous studies have been carried out to clear up the influx mechanism of tissue fluid into the epidermis and the lost cohesion between KCs in acute eczema that leads to spongiosis (6).

The process of spongiosis entails two events: detachment of cells from each other and fluid accumulation between the detached cells. Fluid influx into the malpighian layer occurs because of the osmotic pressure gradient resulting from increased hyaluronan in the epidermis. However, no one can elaborate on the precedence or simultaneity of the two events, i.e. whether the detachment of the cells leads to fluid accumulation, fluid accumulation pushes the cells apart, or the two events occur simultaneously (6).

Spongiosis is induced by early apoptosis of KCs, secondary to cell shrinkage and cleavage of E-cadherin, which is essential in conveying $\mathrm{KC}$ cohesion. It has been shown that the loss of $\mathrm{KC}$ cohesion constitutes the main event in spongiosis formation. Therefore, in spite of being the main driving force of spongiosis formation, fluid influx into the skin is apparently not the primary step, but rather the end result of successive pathogenic events (6).

The use of the Fas protein (CD95) might be the most important way to induce apoptosis by triggering death recep- 


\begin{tabular}{|c|c|c|c|c|}
\hline & Acute $(N=6)$ & Subacute $(\mathrm{N}=\mathbf{2 0})$ & Acute on Top of Chronic $(\mathrm{N}=9)$ & $\mathbf{P}$ \\
\hline Fas receptor & $9.02 \pm 3.22$ & $8.16 \pm 3.12$ & $7.89 \pm 4.95$ & 0.626 \\
\hline Fas ligand & $5.93 \pm 2.39$ & $5.24 \pm 3.06$ & $5.94 \pm 3.86$ & 0.661 \\
\hline
\end{tabular}

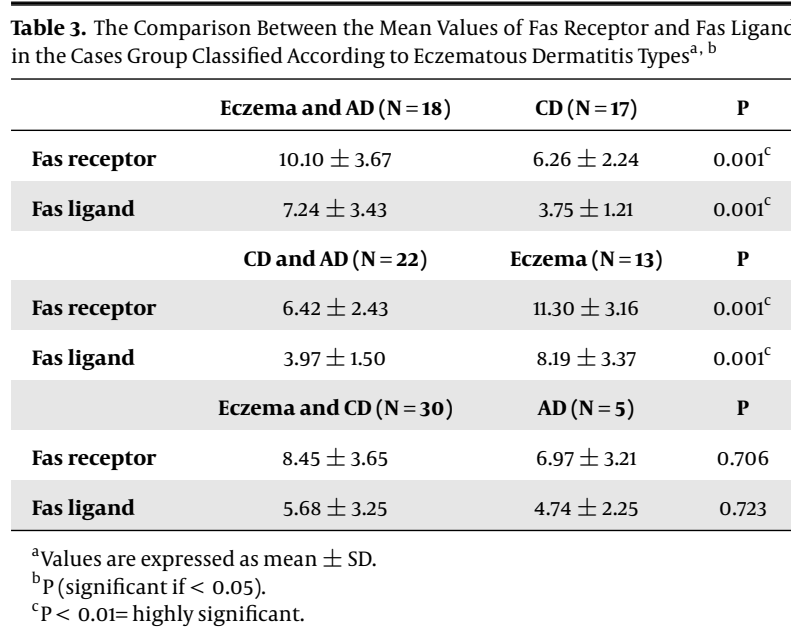

tors, which are a family of transmembrane proteins $(1,10$, 12). Fas activation can induce phosphorylation of nuclear factor of kappa light polypeptide gene enhancer in B-cells inhibitor, alpha ( $\mathrm{I} \kappa-\mathrm{B} \alpha)$, which activates the nuclear factorkappa $\mathrm{B}(\mathrm{NF}-\kappa \mathrm{B})$ pathway, thereby inducing production of interleukin (IL)-8 and TNF- $\alpha$ (13).

FasR stimulation has been suggested as a triggering factor for production of chemokines and cytokines from macrophages, neutrophils, vascular smooth muscle cells, and dendritic cells $(14,15)$. Tumor necrosis factor related-apoptosis-inducing ligand (TRAIL) has also been reported to induce production of chemokines and promote macrophage migration in vitro, although no role has been demonstrated for specific chemokines in this context (16).

Fas stimulation can induce a pro-inflammatory cascade, leading to expression and secretion of multiple chemokines and cytokines. It runs in parallel with events, leading to apoptosis of cells. Cells dying in response to Fas stimulation produce several chemokines [monocyte chemoattractant protein 1/chemokine (C-C motif) ligand 2 (MCP-1/CCL-2), IL-8, regulated on activation, normal T cell expressed and secreted (RANTES)/CCL5 and CXCL1/KC], some of which promote chemotaxis of phagocytes toward the source of these factors (17). Cullen et al. stated, "apoptotic cells may also secrete soluble factors, dubbed "find- me" signals, that can promote chemotaxis of phagocytic cells toward dying cells" (18).

Activated $\mathrm{T}$ cells infiltrating the skin in $\mathrm{AD}$ and $\mathrm{CD}$ upregulate Fas expression by KCs through interferon-gamma (IFN- $\gamma$ ) and induce apoptosis through FasL, which is expressed on the $\mathrm{T}$ cell surface and released into the microenvironment (19). Damage to KCs might occur without cell-cell contact with $\mathrm{T}$ cells, although some T cells apparently migrate between epidermal KCs. However, the precise mechanism of T-cell migration into the epidermis and its link with the apoptosis of KCs are still unknown (20).

These findings support the view that pro-apoptotic pathways are activated in KCs of $\mathrm{AD}$ patients, contributing to at least some of the clinical and histological features of the disease (21). However, spongiosis is limited to the suprabasal epidermis. This is because expression of the cell-intrinsic, caspase-8 inhibitor, c-FLIP, which modulates Fas-FasL signaling, is tightly restricted to the basal layer protecting basal KCs from apoptosis (22).

In this work, we studied the expression of FasR and FasL that are known to induce KC apoptosis and inflammation, in an attempt to identify their intertwined relationship and role in the complex pathogenesis of eczematous dermatitis.

We observed that FasR and FasL were upregulated in the cases group compared to the controls $(\mathrm{P}<0.001)$. However, we could not find in the literature what matches or contradicts these findings. These findings suggest the possible role of the upregulated FasR and FasL in the pathogenesis of eczematous dermatitis. This strongly advocates the importance of further research to explore this point and highlight its value in the future management of such diseases.

We also found that FasR and FasL were upregulated in the eczema cases compared to the cases with the other forms of eczematous dermatitis ( $C D$ and $A D)(P=0.001)$. However, we observed FasR and FasL downregulation in the $\mathrm{CD}$ cases in comparison with the eczema and $\mathrm{AD}$ cases $(\mathrm{P}=0.001)$. No previous studies could be found to match or contradict these results. These findings may suggest that the quantitative amount of Fas expression may alter the clinical picture or modulate the type of eczematous der- 
matitis.

In this study, we did not detect any significant correlation between FasR and FasL with any of the recorded variables, including age and sex, or the disease parameters including stage of disease, site of disease, disease duration, redness, thickness, scratching, and EASI score. This can be explained by the fact that Fas is responsible for the initiation and/or maintenance of eczematous dermatitis without being affected by any variables. Unfortunately, no related studies in the literature could either support or contradict these findings.

Psoriasis induction is promoted by FasR/FasL signaling through activated NK cells. Downstream events, including induction of TNF- $\alpha$ production by KCs and psoriasis histological changes, are prevented by blockage of Fas signaling. Consequently, Fas signaling can be considered an essential early event in the induction of psoriasis via the nonapoptotic pathway (23).

Anti-Fas antibody (ZB4), a monoclonal antibody that blocks Fas-mediated apoptosis, and anti-FasL were both effective in significantly blocking histological changes of psoriasis, including increased epidermal thickness (24). These results can encourage research on the use of anti-Fas antibodies for management of eczematous dermatitis and evaluation of these results. Moreover, this study can open a new era in the management of such groups of dermatological disorders.

According to the results of this work, it can be suggested that the increased expression of FasR and FasL in eczematized lesions is probably involved in the pathogenesis of eczema through several mechanisms including an unchecked pro-inflammatory TNF- $\alpha$ consequence. This result urges to further investigate the issue. In addition, it offers a new therapeutic approach for prevention and treatment of eczematous dermatitis using anti-Fas therapy.

\subsection{Limitations of the Study}

1) The number of the participating patients was small.

2) It was a short-term study.

\section{Footnotes}

Authors' Contribution: Study concept and design: Hanan Rabea Nada, Ahmed M. Nada, and Maha Fathy Elmasry Analysis and interpretation of data: Hanan Rabea Nada, Ahmed M. Nada, and Maha Fathy Elmasry. Drafting of the manuscript: Hanan Rabea Nada and Maha Fathy Elmasry. Critical revision of the manuscript for important intellectual content: Hanan Rabea Nada and Maha Fathy Elmasry. Laboratory work: Laila A. Rashed.
Conflict of Interests: All the authors state that they have no conflicts of interest.

Ethical Approval: The Dermatology Research Ethical Committee (Derma REC) approved the study in accordance with the Helsinki Declaration of 1975, as revised in 1983 (Derma REC; Chairperson: Dr Hoda Rasheed; protocol number: 20/2014; date of approval: 11/12/2014).

Funding/Support: The authors did not receive any fund for this work.

Informed Consent: Written informed consents were obtained from all the participants in the study.

\section{References}

1. Schwarz T. No eczema without keratinocyte death. J Clin Invest 2000;106(1):9-10. doi:10.1172/JCI10438. [PubMed:10880042]. [PubMed Central: PMC314367].

2. Hanifin JM. Evolving concepts of pathogenesis in atopic dermatitis and other eczemas. J Invest Dermatol. 2009;129(2):320-2. doi: 10.1038/jid.2008.252. [PubMed: 18719604].

3. Hanifin JM, Reed ML, Eczema Prevalence; Impact Working Group. A population-based survey of eczema prevalence in the United States. Dermatitis. 2007;18(2):82-91. doi: 10.2310/6620.2007.06034. [PubMed: 17498413].

4. Berth-Jones J. Eczema, lichenification, prurigo and erythroderma. In: Burns T, Breathnach S, Cox N, Griffiths C, editors. Rook's Textbook ofDermatology. 2010. p. 985-1035. doi: 10.1002/9781444317633.ch23.

5. Jones RR. The histogenesis of eczema. Clin Exp Dermatol.1983;8(3):21325. doi: 10.1111/j.1365-2230.1983.tb01774.x. [PubMed: 6883790].

6. Ohtani T, Memezawa A, Okuyama R, Sayo T, Sugiyama Y, Inoue S, et al. Increased hyaluronan production and decreased E-cadherin expression by cytokine-stimulated keratinocytes lead to spongiosis formation. I Invest Dermatol. 2009;129(6):1412-20. doi: 10.1038/jid.2008.394. [PubMed: 19122650].

7. Elgert KD. Immunology: Understanding the immune system. New Jersey: John Wiley \& Sons; 2009.

8. Hotchkiss RS, Strasser A, McDunn JE, Swanson PE. Cell death. N Engl J Med. 2009;361(16):1570-83. doi: 10.1056/NEJMra0901217. [PubMed: 19828534]. [PubMed Central: PMC3760419].

9. Wajant H. Principles and mechanisms of CD95 activation. Biol Chem. 2014;395(12):1401-16. doi: 10.1515/hsz-2014-0212. [PubMed: 25153377].

10. Ashkenazi A, Dixit VM. Death receptors: Signaling and modulation. Science. 1998;281(5381):1305-8. doi: 10.1126/science.281.5381.1305. [PubMed: 9721089].

11. Hanifin JM, Thurston M, Omoto M, Cherill R, Tofte SJ, Graeber M. The eczema area and severity index (EASI): Assessment of reliability in atopic dermatitis. EASI Evaluator Group. Exp Dermatol. 2001;10(1):11-8. doi: 10.1034/j.1600-0625.2001.100102.x. [PubMed: 11168575].

12. Peter ME, Krammer PH. Mechanisms of CD95 (APO-1/Fas)-mediated apoptosis. Curr Opin Immunol. 1998;10(5):545-51. doi: 10.1016/s09527915(98)80222-7. [PubMed: 9794832]

13. Russo MP, Bennett BL, Manning AM, Brenner DA, Jobin C. Differential requirement for NF-kappaB-inducing kinase in the induction of NF-kappaB by IL-1beta, TNF-alpha, and Fas. Am J Physiol Cell Physiol. 2002;283(1):C347-57. doi:10.1152/ajpcell.00166.2001. [PubMed 12055104].

14. Guo Z, Zhang M, Tang H, Cao X. Fas signal links innate and adaptive immunity by promoting dendritic-cell secretion of CC and CXC 
chemokines. Blood. 2005;106(6):2033-41. doi: 10.1182/blood-2004-124831. [PubMed: 15941911].

15. Henry CM, Martin SJ. Caspase-8 acts in a non-enzymatic role as a scaffold for assembly of a pro-inflammatory "FADDosome" complex upon trail stimulation. Mol Cell. 2017;65(4):715-29. doi: 10.1016/j.molcel.2017.01.022. [PubMed: 28212752].

16. Berg D, Stuhmer T, Siegmund D, Muller N, Giner T, Dittrich-Breiholz O, et al. Oligomerized tumor necrosis factor-related apoptosis inducing ligand strongly induces cell death in myeloma cells, but also activates proinflammatory signaling pathways. FEBS J. 2009;276(23):6912-27. doi: 10.1111/j.1742-4658.2009.07388.x. [PubMed:19895579].

17. Ravichandran KS. Beginnings of a good apoptotic meal: The findme and eat-me signaling pathways. Immunity. 2011;35(4):445-55. doi: 10.1016/j.immuni.2011.09.004. [PubMed: 22035837]. [PubMed Central: PMC3241945].

18. Cullen SP, Henry CM, Kearney CJ, Logue SE, Feoktistova M, Tynan GA, et al. Fas/CD95-induced chemokines can serve as "findme" signals for apoptotic cells. Mol Cell. 2013;49(6):1034-48. doi: 10.1016/j.molcel.2013.01.025. [PubMed: 23434371].

19. Trautmann A, Akdis M, Kleemann D, Altznauer F, Simon HU, Graeve $\mathrm{T}$, et al. T cell-mediated Fas-induced keratinocyte apoptosis plays a key pathogenetic role in eczematous dermatitis. J Clin Invest. 2000;106(1):25-35. doi:10.1172/JCI9199. [PubMed:10880045]. [PubMed
Central: PMC517909].

20. Trautmann A, Akdis M, Brocker EB, Blaser K, Akdis CA. New insights into the role of $\mathrm{T}$ cells in atopic dermatitis and allergic contact dermatitis. Trends Immunol. 2001;22(10):530-2. doi: 10.1016/s14714906(01)02004-x. [PubMed: 11574260].

21. Simon D, Lindberg RL, Kozlowski E, Braathen LR, Simon HU. Epidermal caspase-3 cleavage associated with interferon-gammaexpressing lymphocytes in acute atopic dermatitis lesions. Exp Dermatol. 2006;15(6):441-6. doi: 10.1111/j.0906-6705.2006.00428.x. [PubMed: 16689860].

22. Armbruster N, Trautmann A, Brocker EB, Leverkus M, Kerstan A. Suprabasal spongiosis in acute eczematous dermatitis: cFLIP maintains resistance of basal keratinocytes to T-cell-mediated apoptosis. J Invest Dermatol. 2009;129(7):1696-702. doi: 10.1038/jid.2008.438. [PubMed: 19177145].

23. Gilhar A, Yaniv R, Assy B, Serafimovich S, Ullmann Y, Kalish RS. Fas pulls the trigger on psoriasis. Am J Pathol. 2006;168(1):170-5. doi: 10.2353/ajpath.2006.041354. [PubMed: 16400020]. [PubMed Central: PMC1592658].

24. Fadeel B, Thorpe CJ, Yonehara S, Chiodi F. Anti-Fas IgG1 antibodies recognizing the same epitope of Fas/APO-1 mediate different biological effects in vitro. Int Immunol. 1997;9(2):201-9. doi: 10.1093/intimm/9.2.201. [PubMed: 9040002]. 